\title{
The Technical Difficulties and Measures of Horizontal Well Drilling Geologic Tracking
}

\author{
Ji Ling, Wang Qingkui ${ }^{1}$ \\ ('. Graduate School of Petro China Research Institute of Petroleum Exploration \& Development, Beijing \\ 100083,China;2. The third production company, Petro china Dagang oil filed, Cangzhou 061035,China)
}

\begin{abstract}
As one of the effective ways to improve the utilization of reservoir, horizontal well technology has been widely used domestic and abroad. Drilling geological tracking is the key factor of achieving the purpose of drilling horizontal wells and good results. In this paper, the technical difficulties in the process of tracking have been analyzed, and the measures have been put forward. Considering the characteristics of the complex structure and the variable reservoir changes in Dagang oilfield, on the basis of practical tracking experiences on various horizontal wells, the method of calculating the best landing deviation angle of horizontal wells has been put forward, and the response of the landing point in the geological data were summarized. The landing point judging method and well trajectory adjustment time in different geological conditions were also discussed .All the summarized technological measures were fully used in the process of tracking six horizontal wells, all the wells has been successfully drilled and achieved good results.
\end{abstract}

Keywords: Horizontal well, Drilling geological tracking, the best landing deviation angle, Landing point judging, Adjustment Measures

\section{Introduction}

Horizontal well drilling geological tracking is based on the design of the reservoir, using the data of DLWD, geological logging, reservoir prediction, horizon comparison to discover reservoir, estimate the reservoir top depth, analyze reservoir parameter, target design, adjust the horizontal well trajectory in the reservoir and achieve the maximum horizontal drilling rate ${ }^{[1 \sim 4]}$. In the process of horizontal well tracking, there are several technical problems: because the reserves of complex fault block reservoir changes, the optimal window hole angle is hard to determine; limited with a variety of geological data, the window to determine the location is full of difficulties; how to determine the window and how to choose the best time for well trajectory adjustment under different geological conditions.

\section{Calculation of landing deviation angle}

In the implementation of a horizontal well in a reservoir of complex fault block, according to the drilling track, it need to constantly analyze drilling strata development and formation dip, adjust the drilling strata horizontal well drilling inclination angle according to the actual situation, especially the point close to the window and the choice of horizontal inclination is very important. If inclination angle is less than the optimal angle, drill will quickly penetrate the stratum. If inclination angle is greater than the optima angle, drill may not be into the window or into the window quickly out after the formation from the upper piercing.

\subsection{Calculation of theoretical well landing deviation angle}

According to the trace of the target layer, the theoretical well angle is calculated. Horizontal layer (Figure 1):the prediction layer thickness is $\mathrm{h}$, horizontal well drilled layer length is $\mathrm{L}$, in this case, the theoretical angle can be calculated according to the formula (1). For example, when L is $200 \mathrm{~m}, \mathrm{H}$ is $5 \mathrm{~m}$, theoretical well landing deviation angel is $88.6^{\circ}$.Dip layer(Figure 2):,dip calculation: $\alpha$, prediction of layer thickness: $\mathrm{H}$ horizontal well drilled layer length: $\mathrm{L}$, in this case, the theoretical well landing deviation angel can be calculated according to the formula (2). For example: when $\alpha$ is $5^{\circ}, \mathrm{L}$ is $200 \mathrm{~m}, \mathrm{H}$ is $5 \mathrm{~m}$, theoretical well landing deviation angel is $83.4^{\circ}$.

\subsection{Calculate the optimal landing deviation angle}

Complex fault block reservoir formation changes, dip angle and thickness of the reservoir is difficult to predict. According to practical experience, calculating the theoretical well landing deviation angel and drilling with the inclination angle $\beta$ generally less can find objective layer as soon as possible. But the deviation angle is so small, that it is easy to wear out the drilling strata, so we must calculate the best inclination of a horizontal well. Figure 2: horizontal wells $(\beta)$ to the angle of the well drilling, in order to find the objective layer as soon as possible, value should be very large, but when value is larger, the drilling target layer, increasing inclination is difficult, there may be drilled strata. Generally horizontal well drilling in the middle is good to, when large 
enough, as soon as possible into the window, but not too large, when drilling through the objective layer, deviation angle just tune into the layer level is the best, according to the conditions of equation (3), can be calculated value, thus to find the optimum angle of the well horizontal well into the window ( $\beta$ ). For example: when $\alpha$ is $5^{\circ}, \mathrm{L}$ is $200 \mathrm{~m}, \mathrm{H}$ is $5 \mathrm{~m}$, the theory of window angle $\beta$ is $83.4^{\circ}, \varphi$ is $3^{\circ}, \mathrm{R}$ is $10 \mathrm{~m}$, calculation is $5^{\circ}$, the best angle of the well horizontal well into the window is $78.4^{\circ}$.

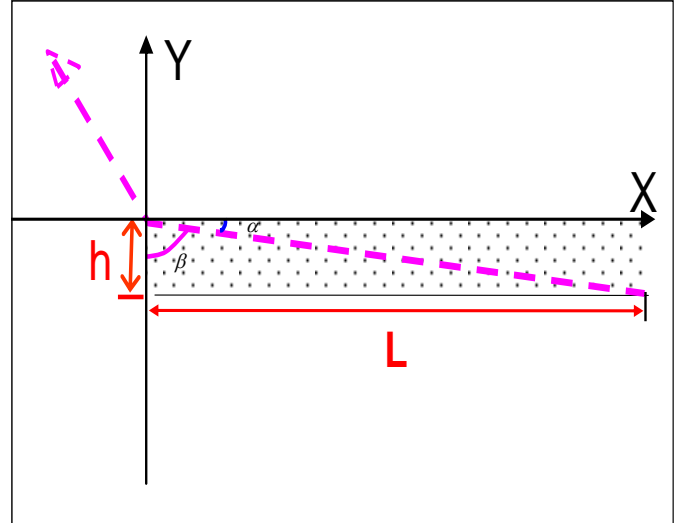

Figure1 Optimal trajectory in the horizontal strata

$$
\beta=\operatorname{arctg} \frac{L}{h}
$$

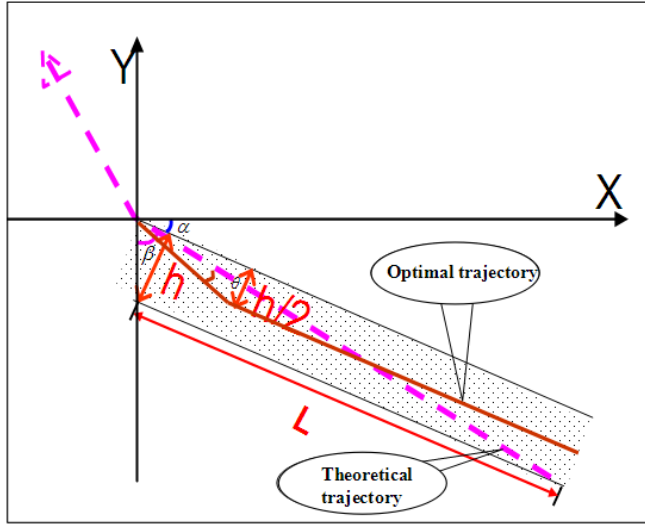

Figure2 Optimal trajectory in the inclined strata

In the formula: $\mathrm{H}$ is the optimal landing deviation angle of the horizontal well; $\mathrm{L}$ is the length of the designed horizontal well penetrating the target layer.

$$
\beta=90^{\circ}-\alpha-\operatorname{arctg} \frac{h}{L+h \cdot \operatorname{tg} \alpha}
$$

In the formula: the horizontal angle of the horizontal well is the optimal landing deviation angle of the window; the dip angle is the angle of formation; $\mathrm{L}$ is the length of the designed horizontal well penetrating the target layer; $\mathrm{h}$ is the formation thickness

$$
\sin (90-\beta-\alpha+\theta) \approx \frac{h / 2}{[(90-\beta-\alpha+\theta) / \varphi] \times R} .
$$

In the formula: $\beta$ is the horizontal wells best window inclination; $\Theta$ is the angle that should be reduced; $h$ is the formation thickness; $\alpha$ is a formation dip; $\varphi$ is the maximum slope of each drill pipe that can be adjusted; $\mathrm{R}$ is the pipe length..

In the process of tracing, we can accurately predict the formation dip angle by using the marker layer, and then calculate the optimal angle of the horizontal well and adjust.

\section{The position judgment}

In the horizontal well drilling geological tracking process, advantages and limitations of several geological data were respective, cutting logging is mainly affected by three factors: first, the collapse effect of the new drilled formation debris judgment; second, the performance of drilling fluid and drilling string combination effect of real debris layer judgment; third, formation interface transition. The drilling time logging is mainly affected by drilling pressure, drilling fluid performance and bit type. Gas logging is mainly affected by the density of drilling fluid and the properties of additives in drilling process. Logging while drilling (LWD) is mainly affected by the factors such as position, borehole, mud invasion, formation heterogeneity and anisotropy. Due to the low vertical resolution, it is difficult to describe the exact position of the horizontal well trajectory in the reservoir. Therefore, we should comprehensively analyze cuttings logging, drilling time logging, gas logging, logging while drilling, three-dimensional seismic data to determine ${ }^{[5-8]}$. The following is based on several horizontal wells tracking experience, summed up the typical characteristics of the window position.

\subsection{Logging response characteristics while drilling}

In the cuttings logging, the content of mud is obviously reduced, and the proportion of the oil and gas shows the proportion of the oil and gas increased in the drop test, and the level of quantitative fluorescence is above 10. In the LWD the drilling time is obviously reduced, which reflects the characteristics of sandstone, drilling time gradually increased, which reflects characteristics of mudstone (Figure 3). 


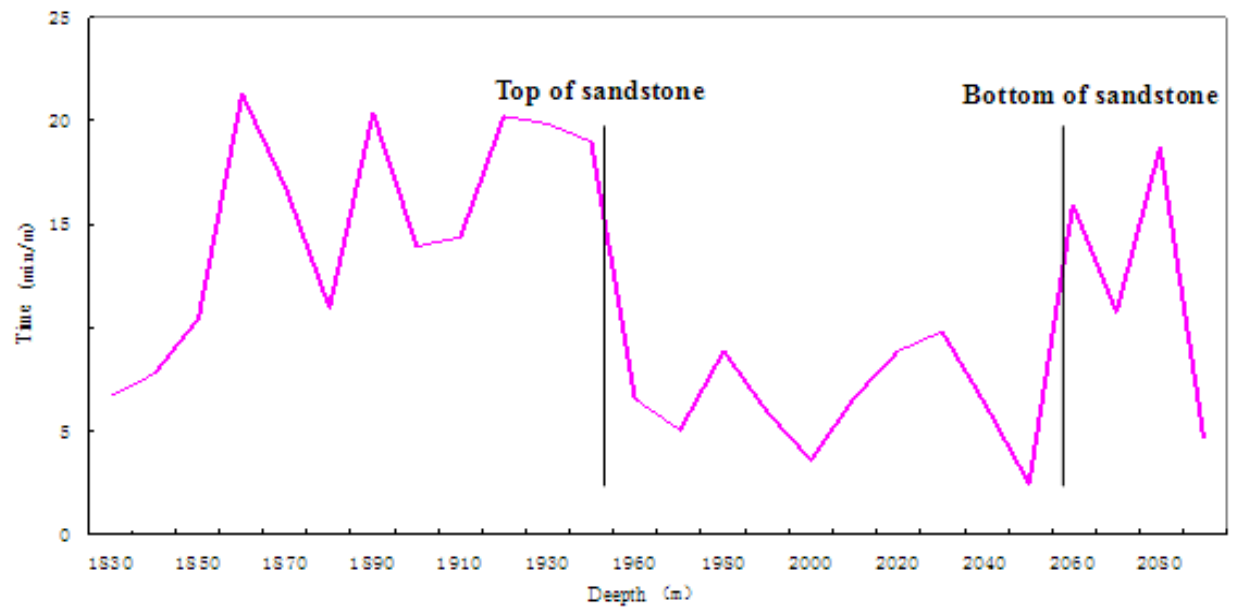

Figure3 Drilling time curve of Well Guan78-3H

In the gas logging well, after entering the window, there are all kinds of components, and the hydrocarbon and the composition of the gas are rising rapidly. When the total hydrocarbon value increases, the drilling time decreases, the hydrocarbon value decreases and the drilling time increases, and the difference between the two is reversed (Figure 4).

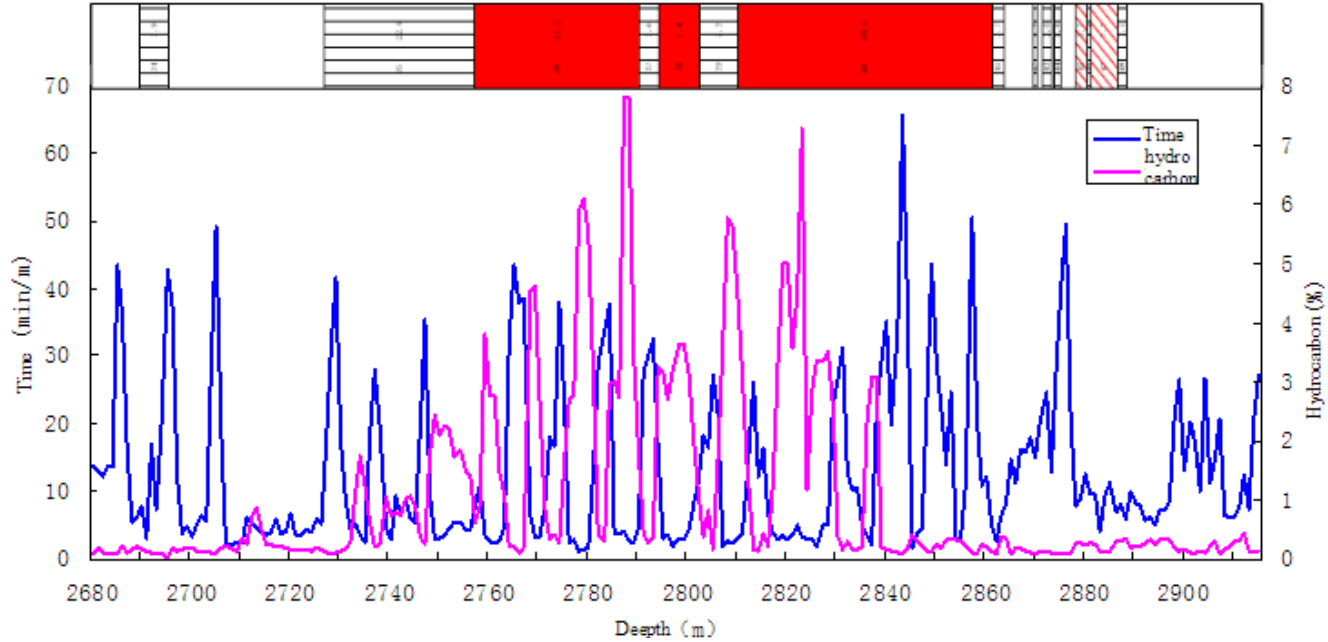

Figure4 Time-Hydrocarbon curve of Well Wang 14-33L

\subsection{Logging response characteristics while drilling}

When the horizontal well is drilled into the window, the logging instrument is close to the reservoir, and the resistivity curve suddenly increases to a maximum and then decreases sharply, which is the polarization angle of the resistivity curve ( Figure 5). The polarization angle is mainly affected by the resistivity difference between the strata, the distance between the instrument and the formation and the relative angle between the center line and the interface. The greater the difference of resistivity between different strata, the more easily the polarization angle. The important sign of the polarization angle phenomenon can be used to judge if the drill is close to reservoir.

The detection radius of resistivity logging is $381.0 \sim 952.5 \mathrm{~mm}$, after the horizontal well is into the window, resistivity detection point goes through mudstone and sandstone along the top of the reservoir drilling trajectory, the measured value is the average of mudstone and sandstone layer resistivity value, therefore, the resistivity value of horizontal well is much lower. The radius of the gamma ray logging is $228.6 \sim 304.8 \mathrm{~mm}$, which is is smaller than the resistivity detection depth, the gamma curve of mudstone and sandstone lithologic interface response is relatively objective and true ${ }^{[9-11]}$, so in the window point judgment should make full use of the natural gamma curve. 


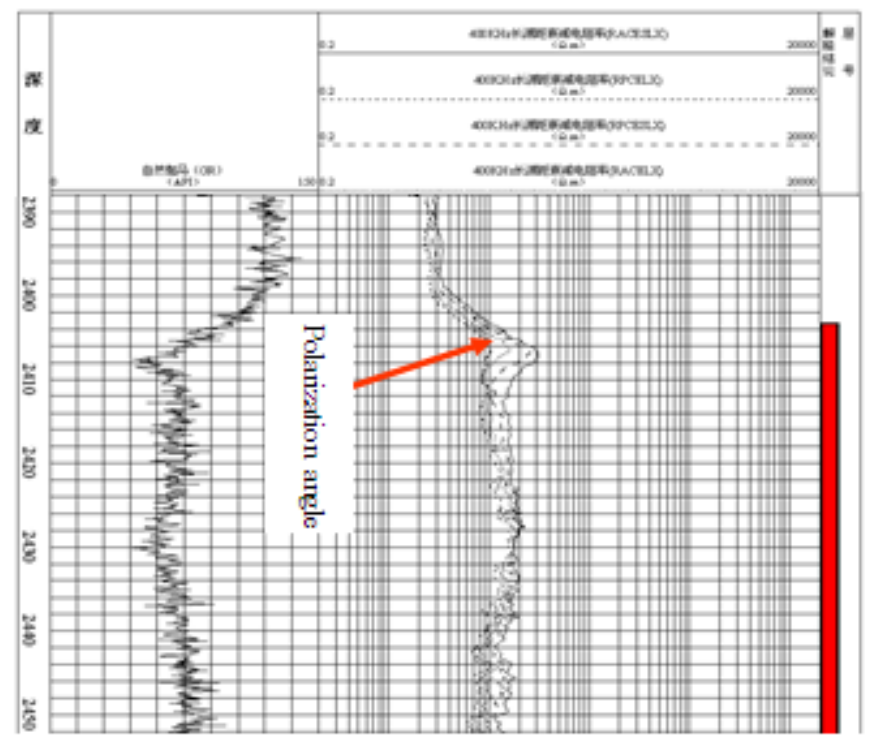

Figure 5 Polarization angle

\section{Adjustment Strategies under Different Circumstances}

Dagang Oilfield reservoir is a complex fault block reservoir with complex structure.In the horizontal drilling process, it may encounter a variety of complex situations, when the drilling meets faults, edge and bottom water reservoir we should take different measures.

\subsection{Drilling fault}

If the fault is developed between the wellhead and the horizontal section, the position of the marker layer can be changed after drilling the fault, which increases the difficulty of field tracking. In this case, we should focus on the detailed description of the local structure,fault development in the horizontal well, and the interval of the structure is less than $5 \mathrm{~m}$. In the drilling process of tracking, drilling in fault area prediction, we should encrypt logging sampling interval, increase the contrast of power measurement, and add real-time drilling trajectory to seismic loading body,compare with the known wells, eventually adjust the breakpoint position. Combined with the depth of the well known breakpoint, calculate the formation dip that is close to actual formation dip. Because of the influence of the fault dip on the fault near the fault, it is necessary to drill through the section for a certain distance, analyze and calculate a reference dip angle, and adjust the trajectory according to the formation condition.

\subsection{Edge water reservoir}

For edge bottom water reservoirs, the position should be designed to accurately judge the depth of the oil-water interface, the window should be chose $1 \sim 3 \mathrm{~m}$ in the reservoir below the top surface of the design, the outlet position should have a certain distance from the oil-water interface, which is thick enough to avoid water. In the process of tracking, trajectory is controlled in a window that covers radius $*$ swing swing radius radius * level long box, the front and back window radius is generally controlled within $5 \mathrm{~m}$, up and down swing radius is controlled in generally $1 \sim 3$, sometimes less than $1 \mathrm{~m}$,swing radius is generally controlled within $10 \mathrm{~m}^{[6]}$. For the height of the smaller oil reservoir, the reservoir in horizontal section has not yet settled, according to the comprehensive prediction, with a certain angle gradually adjusted, in order to ensure the oil drilling to settle, ensure a large enough water avoidance thickness.

\section{Conclusion}

1) In the horizontal well drilling tracking process, calculate the optimal angle of the horizontal well and timely adjustment of the trajectory can ensure that the location of the window is combined with a variety of information to accurately judge. When the drill bit is into the reservoir, comprehensive logging parameters would have significant changes: when drilling is down; mudstone cuttings in sandstone decrease, the proportion of debris increases; gas logging value is component, while the rapid rise and total hydrocarbon values increase. LWD curve shows that resistivity curve is closer to the interface, the resistivity value is high, and the polarization angle phenomenon will occur, when the GR curve goes through from mudstone to sandstone formation, it is more close to the interface and the influence is small with a lower GR.

2) Under different drilling conditions, the focus is different. If the forecast window is into drilling fault, and the fine description of structure is the focus, and in the process of tracking encryption logging and electric 
measurement interval, fine stratigraphic correlation determines the breakpoint location and dip. If the horizontal wells drill into edge water reservoir, in the process of tracking, the key is to ensure the drilling rate is under the premise of controlling bit vertical depth, ensure the height of water avoidance of certain.

\section{Reference}

[1]. Niu Xiangyu.Optimization design technology of fractured horizontal well in low permeability reservoir[J].Journal of Oil and Gas Technology,2009,31(2): 120 122.

[2]. Yang Yaozhong,Chang Feng,etc.Research on the development of horizontal well in thin oil reservoir with high permeability in Pinghu oil and gas field[J].Petroleum Exploration and Development,2003,30 (1): 90 92.

[3]. Sun Jinhao.Research and application of horizontal well geological guidance and interpretation technology[J].World Well Logging Technology,2010,(4): 14 19.

[4]. Qin Zongchao,LiuYinggui etc.Application of geosteering technology in complex fluvial oil field in Caofeidian 11-1 oilfield as an example[J].Petroleum Exploration and Development,2006,33 (3): 378 382.

[5]. Lu Zhiyong.Study on the method of geological tracking and adjustment of horizontal well trajectory: a case study of Xin 109 fault block Ping 7[J].Journal of Oil and Gas Technology,2012,34 (9): 231 234.

[6]. Zhu Weihong,Niu Jingui etc.Geological design and tracking method of horizontal well in complex oil and gas reservoir[J].Drilling and Production Technology,2010,32(1): 111-116.

[7]. Feng Xiangzi,Li Da.Analysis and correction of influence factors of horizontal well logging while drilling[J].Science Technology and Engineering,2011,11(8).

[8]. Yang Yuru,Li Jianping etc.Horizontal well in Bozhong 25-1 oilfield drilling reservoir prediction and Optimization Management[J].Well Testing 2007,16(4): 62-65.

[9]. Wang Wei,Yin Kai.Analysis of abnormal shape of well logging curve while drilling in high angle and horizontal wells[J].China Offshore Oil and Gas,2009,21 (1): 27-30

[10]. Chen Song,Ren Baosheng etc.Logging research and example analysis of horizontal well while drilling[J].Chinese Journal of Engineering Geophysics,2009,6(6): 787-791.

[11]. Zhou Cancan,Wang Changxue.Review of horizontal well logging interpretation technology[J].Progress in Geophysics,2006,21(1): $152-1$ 